42 | 2012

La communication, dimension oubliée de l'intelligence économique

\title{
Le document numérique : un nouvel équipement politique de la mémoire sociale?
}

Pascal Robert et Nathalie Pinède

\section{OpenEdition}

\section{Journals}

Édition électronique

URL : http://journals.openedition.org/communicationorganisation/3948

DOI : 10.4000/communicationorganisation.3948

ISSN : 1775-3546

Éditeur

Presses universitaires de Bordeaux

Édition imprimée

Date de publication : 1 décembre 2012

Pagination : 191-202

ISBN : 978-2-86781-772-4

ISSN : $1168-5549$

Référence électronique

Pascal Robert et Nathalie Pinède, « Le document numérique : un nouvel équipement politique de la mémoire sociale? », Communication et organisation [En ligne], 42 | 2012, mis en ligne le 01 décembre 2014, consulté le 04 mai 2019. URL : http://journals.openedition.org/

communicationorganisation/3948; DOI : 10.4000/communicationorganisation.3948 


\title{
Le document numérique : un nouvel équipement politique de la mémoire sociale?
}

\author{
Pascal Robert ${ }^{1}$, Nathalie Pinède ${ }^{2}$
}

La mémoire sociale de nombreuses sociétés dépend d'outils qui permettent de donner corps aux opérations du penser/classer. Ces outils, on peut les appeler à la suite de J. Goody (Goody, 1979) des technologies intellectuelles. En leur cœur, parce qu'elles reposent toujours sur un support et proposent toujours une mise en forme, on retrouve inévitablement le document. Ces technologies intellectuelles permettent d'effectuer une opération fondamentale : celle de la décontextualisation dans l'espace et le temps. Une opération, nous le verrons, aux effets politiques considérables. Le passage des supports traditionnels, et notamment du papier, au support numérique déplace les enjeux techniques et politiques de cette opération de décontextualisation. Nous proposons d'explorer les modalités concrètes et les effets politiques de ce déplacement, entre continuités par rapport à des modalités traditionnelles et ruptures induites par les spécificités du mode numérique et de la mise en réseau.

Dans un premier temps, nous explorerons les dimensions liées à l'opération de décontextualisation, en comparant deux formes d'autonomie documentaire, celle du papier et celle générée par le numérique. Ce qui nous permettra dans un deuxième temps d'explorer deux modes en articulation, logistique et document, l'une influant sur l'autre et réciproquement. Cette mise en évidence de ce double mouvement nous amènera à poser, à partir de cette problématique du numérique et du document, trois perspectives d'infléchissement autour du triptyque politique/mémoire/société.

1 Pascal Robert est Professeur en sciences de l'information et de la communication à l'ENSSIB (Ecole nationale supérieure en sciences de l'information et des bibliothèques) et chercheur au laboratoire ELICO (Equipe de recherche de Lyon en sciences de l'information et de la communication) ; pascal.robert@enssib.fr

2 Nathalie Pinède est Maître de conférences à l'université Michel de Montaigne Bordeaux 3 et chercheur au laboratoire MICA (Médiations, Information, Communication et Arts) ; nathalie.pinede@iut.u-bordeaux3.fr 


\section{Décontextualisations : du papier au numérique}

C'est l'inscription de données sur un support qui permet à l'information, rapportée à un processus communicationnel, de s'affranchir de contraintes, comme celles imposées par la synchronie du mode oral, et d'introduire de la distance, dans le temps et/ou dans l'espace. À ce titre, le support, quelle que soit sa forme matérielle et quelle que soit la technique d'enregistrement de données, permet la transmission, en tant que « medium » (Chabin, 2004) et assure une fonction mémorielle. Toutefois, si tout support permet cette décontextualisation et donne à l'information son statut documentaire, les formes d'autonomie générées varient grandement en fonction de la nature du processus et des « matériaux » utilisés.

\section{L'autonomie intrinsèque du document papier}

Il nous faut dans un premier temps revenir à l'opération de décontextualisation pratiquée par le document traditionnel. Ce dernier, en effet, la rend possible grâce à son autonomie pratique liée à son support principal. Celui-ci, la feuille de papier notamment, en fait un objet en soi, circonscrit, fini, dont on peut faire le tour avec les yeux, le balayer globalement ou se focaliser sur telle ou telle portion. On peut le prendre en main, à proprement parler le manipuler. La feuille, soulignons-le, car il s'agit d'un trait fondamental, est première ; elle est volante a-t-on remarqué depuis longtemps et suffisamment pour pouvoir être reliée dans ces outils d'accumulation que sont les livres. Le livre relie et tient par là même les choses qu'il contient. Les comptables le savent bien qui enserrent leurs comptes justement dans des livres afin qu'ils ne s'en échappent pas de manière intempestive : la forme livresque assume cette confiance dont la seule feuille, volage, ne peut les assurer. Mais à son tour le livre constitue un objet autonome, sans être forcément indépendant puisqu'il est (à nouveau, mais autrement) relié aux autres livres par un réseau bibliographique souvent dense.

Par ailleurs, son support, dès lors qu'il est suffisamment léger et solide, rend ce document transportable tant dans l'espace que dans le temps (monumental - à l'instar du code Hammourabi gravé sur une stèle - il n'autorise alors qu'un transport dans le temps). Même les tablettes d'argiles ont su traverser la longue durée comme aurait dit Braudel, par milliers d'exemplaires. J. Goody a montré, après les archéologues, qu'elles étaient aussi des outils de mémoire qui permettaient de gérer l'état en voie de constitution, dans son extension spatiale comme dans son évolution historique. La bibliothèque est traditionnellement le parangon de ce type d'outil de décontextualisation. Ainsi Ch. Jacob (Jacob, 1991) a-t-il souligné que, un peu plus près de nous, la bibliothèque d'Alexandrie n'était pas possible sans un lourd travail - politique - de réquisition de rouleaux, d'échanges ou d'achats sans lequel l'on n'aurait pas pu constituer le fonds : il a fallu une vaste circulation dans l'espace de l'est de la Méditerranée sur un temps long 
pour construire cette accumulation. Car c'est bien de cela qu'il s'agit, comme l'a montré B. Latour (Latour, 1989) : cette autonomie offerte par le support est elle-même mobilisée dans des réseaux et des centres d'accumulation qui relèvent pour la plupart du pouvoir politique et/ou économique. Plus globalement, et pour nous introduire à une modernité dont nous ne sommes pas sortis (Robert, 2011), c'est ce dispositif de saisie documentaire qui permet de construire la logique de la discipline telle que l'a mise en évidence M. Foucault : les hommes et les choses sont captés nous dit Foucault dans des processus d'écritures, que ce soit à l'école, à l'armée, à l'hôpital ou à l'usine par les techniques précises de l'examen (listes, tableaux, etc.) (Foucault, 1975).

Qui plus est, le document a été muni de ce que, en nous appuyant sur les travaux d'E. Souchier (Souchier, 1996), l'on peut appeler ses signes d'énonciation éditoriale qui lui offrent une configuration sémiotique reconnaissable (on peut, avec un minimum d'entraînement assez facilement et à vue d'œil, c'est-à-dire avant la lecture elle-même, savoir s'il s'agit d'un document juridique ou religieux). Autrement dit, le document n'est pas seulement autonome parce que son support le rend déplaçable dans l'espace et le temps, mais aussi parce que son inscription, sa graphie et ses modes d'organisation et de structuration lui assurent également une forme d'autonomie, celle d'une véritable énonciation documentaire. L'écriture, en effet, rompt avec les conditions d'énonciation de l'oralité - quitte à travailler, dans le texte littéraire notamment, à les réintroduire par quelques déictiques par exemple. Elle assure l'autonomie intellectuelle relative du document : non qu'il reste forcément lisible partout et de tout temps, mais dans l'espace - temps singulier d'une culture - celle des différentes entités politiques du Moyen Orient antique, de l'empire romain, de notre moyen âge etc. On peut, ultérieurement, apprendre à le déchiffrer et à en comprendre le sens.

\section{L'autonomie par délégation du document numérique}

Aujourd'hui, le document numérique subit non pas un processus de dématérialisation, mais bien un changement de régime de matérialisation (Robert, 2004) qui déplace, voire modifie considérablement les modalités de l'opération de décontextualisation. C'est pourquoi nous allons, dans un second temps, revenir sur le document numérique comme nouvel instrument politique de la mémoire sociale.

En devenant numérique, le document, en effet, passe d'un support relativement stable et circonscrit à un support plutôt instable et difficile à circonscrire, puisqu'il s'agit d'un objet-système (l'ordinateur) qui est déjà un réseau en soi et qui peut être également articulé directement à des réseaux externes d'échelles variables, du local au global (mondial). Car les TIC sont intrinsèquement des technologies de gestion de flux (électroniques/ informationnels) internes contrôlés/coordonnés susceptibles de contrôler/ 
coordonner des flux informationnels externes (Robert, 2005, p. 110). Cela ne signifie pas l'absence de matérialisation, mais une transformation profonde du mode d'existence matériel du support documentaire : le document, nous y reviendrons, est ainsi inséré, inclus dans une logique, ou plutôt une véritable logistique, dont il ne peut être extrait (sauf sous forme papier, à l'ancienne si l'on veut). Autrement dit, il dépend non plus d'une matérialisation comme objet individualisé, mais comme partie prenante d'un système d'objets (qui relie les objets-systèmes que sont les " ordinateurs " sous toutes leurs formes - PC, smartphones, tablettes, etc.). Cet environnement là devient son milieu de vie, c'est-à-dire de création, de pérennisation, de transformation et de disparition ; c'est lui qui lui assure les formes singulières de son apparition, de sa manifestation à nos sens, à notre perception.

Ainsi, parler de dématérialisation à propos du numérique est-il singulièrement excessif et trompeur. Car avec une telle notion, d'une certaine manière il n'y a plus rien à penser dès lors qu'il n'y aurait plus rien à voir. Les signaux numériques, dès lors qu'ils échappent au spectre de notre visibilité, entreraient ainsi dans le registre de cette supposée dématérialisation : c'est replier la matière sur le visible, ce qui semble pour le moins réducteur. Il ne s'agit donc que de l'illusion de la dématérialisation. La notion de support demeure et assure le processus de trace et de mémoire (les données numériques sont enregistrées, selon des procédés optiques ou magnétiques, sur de multiples supports de stockage). Elle permet également l'affichage des données multimédias et leur manipulation par les interfaces. La miniaturisation des dispositifs de mémorisation comme d'affichage ne doit pas plus être confondue avec un mouvement de dématérialisation en ce sens que, même petit, c'est-à-dire prenant de la distance avec le registre de la visibilité lié à nos sens (au rapport yeux/mains) elle ne cesse d'exister sous forme matérielle. Le petit peut disparaitre de la portée de notre regard sans cesser d'être matériel pour autant. Sauf à confondre encore une fois notre registre de visibilité avec la matérialité, tout ce qui lui échappe tendant à devenir ipso facto suppose-t-on immatériel.

En revanche, cette illusion de la dématérialisation, cette fausse transparence et virtualité des données numériques résulte pour partie de la rupture sensible entre l'enregistrement des données et la perception du message. Y. Jeanneret et $\mathrm{E}$. Souchier ont souligné voilà déjà quelques années (Jeanneret et Souchier, 2002) que l'informatique assurait un découplage fonctionnel de ce que le papier intègre : en effet, lorsque l'on écrit sur une feuille de papier à la main, le geste même du tracé de l'écriture, qui en assure l'affichage, est en même temps sa mémorisation. Linformatique, à la suite de la première mécanisation de l'écriture par la machine à écrire (Gardey, 2008), qui découplait déjà le lieu de la frappe de celle de l'affichage et de la mémorisation, en vient à circonscrire trois espaces nettement distincts sur l'ordinateur : celui de la saisie (le clavier), celui de l'affichage (l'écran) et celui de l'enregistrement (la mémoire). Les trois, quoique condensés, restent séparés, même dans un smartphone. C'est 
dans cet écart que se glisse et se manifeste la supposée dématérialisation. Autrement dit, certains considèrent comme matériel le document où les trois fonctions sont repliées sur un même geste et comme moins matériel le document qui les distingue tout en les articulant dans l'objet système que, dès lors, il constitue.

Ce nouveau régime de matérialité pour le document numérique suscite également de nouvelles formes d'autonomie pour celui-ci, différentes de celle induite/possédée par le document papier. La non accessibilité « sensible » aux données enregistrées (elles existent mais échappent à nos sens, et seraient-elles « lisibles », elles demeureraient inaccessibles à notre appréhension du fait de leur codage binaire), nous rend dépendants, au-delà des supports de stockage et de mémorisation, aux supports de restitution (de type écrans) ainsi qu'aux processus de conversion intermédiaires qui vont nous « traduire " les données en signes enfin compréhensibles. Là encore, la rupture générée par le numérique au plan de l'articulation support/message modifie le modèle de l'autonomie pour le document et n'est pas sans conséquences au plan de son appréhension/utilisation, notamment dans une perspective de rationalisation, voire de contrôle (implicite/explicite).

Où l'on passe d'une autonomie fondée sur la transportabilité du support comme objet individuel et séparé à une toute autre autonomie qui est celle de l'autonomie de la machine elle-même. Car nous déléguons à cette machine quelque chose qui nous échappe, cette puissance de calcul (ce véritable automatisme) qui lui permet de faire ce que nous ne pouvons/savons pas faire (fonctionner à l'échelle électronique, à une vitesse qui défie nos sens, etc.). Ce qui ne signifie pas que nous ayons perdu tout contrôle, mais que nous avons de fait concédé une part non négligeable à la machine elle-même, au niveau de l'objet-système (l'ordinateur) comme au niveau des macro-systèmes techniques des réseaux (Gras, 1993 ; Robert, 2005). Cette autonomie par délégation est considérablement différente de l'autonomie intrinsèque des supports précédents. Ses conséquences sont elles aussi nouvelles, autrement dit, nous commençons à peine à les penser. Nous présenterons deux pistes de réflexion/recherche complémentaires.

\section{Logistique, document et politique}

« Logisticisation du document » et « documentarisation de la logistique »

La « logisticisation du document »

Comme nous l'avons vu plus haut, le document numérique n'est plus forcément stable, il s'intègre dans une gestion de flux documentaires. L. Merzeau souligne ainsi que « les projets d'archivage du web montrent [...] que les institutions mémorielles doivent désormais concevoir des systèmes ouverts, capables d'enregistrer l'information avec son instabilité. Ayant à gérer 
des corpus où les unités se renouvellent et s'interpénètrent constamment, la mémoire est vouée à l'hyperarchive. Elle n'a plus pour fonction de contenir un stock, mais seulement d'en garantir un accès documenté et différé » (Merzeau, 2006). À ce titre, le document numérique acquiert une indéniable dimension logistique, opération que nous qualifions de «logisticisation du document». Auparavant on intervenait pour renforcer la stabilité du document (par un sceau par exemple), aujourd'hui il faut intervenir pour stabiliser et fermer l'accès au document (comme par exemple la production et le verrouillage d'un document PDF). Auparavant on intervenait pour insérer le document dans un réseau, aujourd'hui il faut (souvent) intervenir pour ne pas insérer un document dans un réseau.

Cette « logisticisation » du document intervient à un double niveau : tout d'abord, dans ce phénomène de découplage des supports que nous avons évoqué plus haut. En effet, cette rupture entre le codage numérique de l'information et notre accessibilité à cette nature d'information entraîne une délégation et une délocalisation mémorielles et, simultanément et paradoxalement, une relocalisation multiple des lieux et espaces de stockage des données, (souvent en avatar papier, d'ailleurs). Dans l'environnement numérique, la notion de document " original » (originel) disparaît ; on ne cesse d'enregistrer et de ré-enregistrer, sur un même disque dur en des lieux multiples d'une organisation hiérarchique ${ }^{3}$, sur des mémoires amovibles (clef USB, CD-ROM, etc.), avec des formats différents, etc. Or, la persistance du document numérique dépend de la capacité de son support à résister à la dégradation physique (qu'elles qu'en soient les modalités) et de sa logistique (a minima de la disponibilité d'un lecteur) car elle va également passer par la reproductibilité et le stockage qui, à grande échelle, ont un coût considérable (loin de l'idéologie du gratuit). Ce qui ne va pas sans poser de nombreux problèmes, tant au niveau de la pérennité des supports que de l'équipement technique permettant la lecture des donnés numériques.

Dans le même ordre d'idées, on peut également signaler les documents en mode révision qui produisent des images évolutives d'un document à différents stades de création dans un processus collaboratif. Le document circule entre les auteurs, pour des amendements et écritures successifs, et cette logistique génère de multiples versions, à la fois inscrites et dynamiques, qui multiplient les images en miroir du document.

À un deuxième niveau, le document numérique est aujourd'hui un document quasi-naturellement " connecté », presque à notre insu : l'immense majorité des terminaux sur lesquels il est créé sont des terminaux reliés à des réseaux, des systèmes dans lesquels il va s'insérer d'un seul clic, que ce soit via le courrier électronique ou via le web dans ses multiples dimensions (traditionnel ou

3 Un exemple symptomatique étant les documents que l'on reçoit par courriel, et que l'on n'enregistre pas forcément dans la classification de notre ordinateur : notre messagerie électronique devient ainsi une mémoire vivante mais anarchique de notre activité. 
web 2.0). Le document numérique est donc à la fois sur-stocké et en même temps inscrit dans une logique de flux, deux forces qui le rendent multiple et insaisissable. C'est à une véritable inflation et dispersion documentaires à laquelle on assiste, brouillant les pistes classiques du document papier : le document numérique est partout et parfois « nulle part ».

\section{La documentarisation de la logistique}

De façon symétrique, la logistique acquiert de son côté une dimension documentaire (ce que nous appelons la «documentarisation de la logistique ») : pas de logistique physique (déplacement d'objets ou d'hommes) sans son redoublement informationnel, c'est vrai depuis longtemps (Chandler, 1988) et encore plus aujourd'hui (comme par exemple tout ce qui est de type GED - gestion électronique de documents - dans les entreprises ou les administrations) ; il en va même de la création d'une véritable logistique informationnelle documentarisée, que cela soit pour une commande sur un site tel qu'Amazon ou la possibilité de développer un véritable effet documentaire par la traçabilité qu'offrent les réseaux sociaux.

Ce document aux formes multiples, aux supports cachés mais dynamiques, foncièrement inscrit dans des flux que nous ne maîtrisons pas toujours, génère de nouvelles productions documentaires, comme les métadonnées, chères au Web sémantique, ou de façon plus informelle et diffuse, sous forme de traces. Ce processus logistique peut s'accompagner de normes et procédures contraignantes, comme dans les systèmes de type workflow (Pinède, 2006). Mais de façon plus souterraine, la mise en réseau de documents, d'informations numériques ne cesse de générer des marquages accompagnant les mouvements et flux, de nouvelles formes d'empreinte documentaire, plus ou moins visibles. "Alors que le signe tendait idéalement vers le partage d'un sens par le plus grand nombre (code), la trace vise à calibrer au plus près des renseignements sur mesure (signature)» (Merzeau, 2010).

Ces formes de documentarisation participent d'un processus de ré-ancrage d'une logistique qui n'est intangible qu'en apparence : nombre de traces ne cessent d'être dérivées des flux de données, que cela soit par le biais de nos navigations sur les différents sites et réseaux sociaux, ou encore par la construction à facettes de notre identité numérique qui se dégage de notre présence fragmentée sur le Web.

\section{Les conséquences politiques}

Ce double mouvement engendre trois conséquences politiques de fond.

La " gestionnarisation » de la société

Il nourrit un processus plus vaste qui affecte toute notre société et dont les TIC sont en quelque sorte le fer de lance logistique, celui de la " gestionnarisation » de la société (Robert 2004, 2009 et 2011), soit un 
processus par lequel des problèmes qui n'avaient jamais été identifiés comme relevant de la gestion sont désormais saisis de manière privilégiée au travers de cette catégorie et deviennent dès lors susceptibles de recevoir une réponse elle-même gestionnaire, toujours basée sur un traitement informationnel automatisé. Cette gestionnarisation se décline même aujourd'hui sous la forme d'une auto-gestionnarisation rendue possible par l'usage des réseaux sociaux dans laquelle l'internaute participe à son propre dévoilement et à sa propre traçabilité.

\section{Le " glissement de la prérogative politique "}

Il alimente également la logique du "glissement de la prérogative politique » (Robert, 2005) dès lors que la question de la gestion de ces mémoires relève de moins en moins de l'Etat mais de plus en plus d'instances privées, dès lors que la mise en mémoire est moins envisagée comme une question politique que comme une question économique, voire plus du tout comme une question mais seulement à travers la supposée neutralité de la réponse réseautique (Google et Facebook ne seraient pas perçus comme des entreprises mais comme des instances du réseau).

Google serait ainsi de manière privilégiée une entreprise de mémoire alors que Facebook serait une entreprise du flux. Google, avec Google books, G-maps, G-image (ou son nouveau projet de muséographie) s'inscrit de facto dans une logique qui, progressivement, est passée du moteur de recherche qui brasse une mémoire vive, à court terme, de sites ou blogs en chantier permanent à un politique mémorielle de long terme qui s'appuie sur les acquis de la culture longue de l'écrit et de la peinture. Ce par quoi l'entreprise américaine se légitime par une réassurance auprès de la culture cultivée. $\mathrm{Ce}$ en quoi elle développe - et nous reprenons le mot volontairement - une véritable politique culturelle qui l'amène à la fois à entrer en concurrence, parfois rugueuse, avec les instances étatiques (comme ce fut le cas avec la BNF - Jeanneney 2006) ou à nouer des alliances qui introduisent cette entreprise à jouer bien plus que le rôle d'un mécène puisque Google crée des espaces d'expression qu'elle maîtrise.

De son côté, Facebook apparaît plutôt comme une entreprise du flux, de ce qui passe et transite, de l'éphémère, qui néanmoins permetl'auto-accumulation par l'internaute d'informations qui le concernent et qui constitue une mémoire vive ou mémoire courte. Auto-dévoilement de la personnalité ou à tout le moins du comportement, transparence nouvelle qui est aussi tout en même temps une surveillance qui procure un pouvoir à proprement parler politique de redéfinition des catégories légitimes (par quelque folksonomie ?), qui seront de moins en moins la prérogative de l'État et de plus en plus celle d'une entreprise à vocation mondiale. L'enjeu futur pour chacune d'entre elles est ainsi clair : pour Google, renforcer sa logique d'entreprise de mémoire tout en se positionnant autant que faire se peut comme entreprise de flux ; 
pour Facebook, passer d'une entreprise de flux (en tenant la position face à la concurrence localisée), de mémoire vive à une entreprise de la mémoire longue.

Vers un risque mémoriel majeur?

On peut enfin se demander si l'on ne passe pas ainsi d'une mémoire sociale équipée ponctuellement par la technique à une technique qui devient la forme dominante de la mémoire sociale. Dès lors une panne de réseau devient ipso facto également une panne de mémoire, non seulement professionnelle, transactionnelle mais également relationnelle/domestique puisque même le nouveau régime numérique de nos conversations est enregistré (SMS, mail ou autre). Ce qui est poser, à nouveaux frais, la question politique du risque informationnel majeur (Robert 2005), comme risque mémoriel majeur.

\section{Conclusion}

Le mode d'existence du document numérique se révèle singulièrement différent de celui du document papier, sa manière de "faire mémoire " également, notamment dans sa relation au politique.

Le document papier est depuis fort longtemps maintenant (et d'autres supports avant lui bien évidemment) un vecteur privilégié d'une mémoire sociale que l'on a envie de qualifier d'administrative, une mémoire de notaires (pour les actes privés), une mémoire bureaucratique (au sens Wébérien) porteuse de la rationalité étatique (pour les actes publics) comme celle de la grande entreprise (et les deux, que l'on oppose souvent sont en fait très proches) et de leur capacité à discipliner les corps à travers ces instances institutionnelles de l'école, de l'administration, de l'hôpital et de l'usine. Cette mémoire, de long terme, qui n'exclut pas une mémoire orale/sociale non écrite de court (voire moyen) terme, est " paperassière ${ }^{4}$ ». Elle relève de la logique de l'archive, d'un archivage de nos parcours de vie, mais seulement à travers ces points d'ancrages institutionnels qui laissaient, par ailleurs, une relative liberté, ou à tout le moins la possibilité au contrôle social de s'exprimer par d'autres voies (à l'instar du regard ou plutôt de l'entre-regard villageois ou du quartier).

Le document numérique ne disparaît pas en tant que document, c'est-à-dire en tant que porteur de la fonction documentaire : comme capacité à stabiliser le « ce dont il est question ", à le fermer et tout en même temps à le certifier conforme, à le marquer d'un sceau de légitimité. Mais les modalités de sa manifestation se transforment. Car le document n'existe plus d'évidence comme un objet singulier, mais pris dans une logistique dont on ne peut (pas facilement) l'extraire : auparavant le document, autonome, circulait ; aujourd'hui, il circule et voit son autonomie saisie et redéfinie par cette circulation. Son autonomie « objectale » si l'on veut n'est plus évidente, mais

4 Au point que l'on confond volontiers la bureaucratie, qui est un mode d'organisation, avec le document papier qui manifeste la nature écrite de ce mode d'organisation et son mode de fonctionnement. 
à construire, à reconstruire par des procédures qui permettent de redéfinir ce qui est d'abord un élément du flux comme un document (stable, stabilisé/ stabilisant). Ce qu'il perd là (et qu'il faut donc gagner à nouveau par une intervention spécifique) il le gagne en revanche dans son insertion dans une logique de système qui lui permet de participer à sa configuration et à sa dynamique. Ce qui assure à ce système cette autonomie de délégation grâce à laquelle ces macro-systèmes techniques produisent des effets de mémoire qui échappent largement à notre contrôle, " notre » renvoyant à la vieille figure du citoyen. Autrement dit, ne s'agit-il pas d'une technicisation de la mémoire sociale qui risque de s'affranchir de la citoyenneté ?

\section{BIBLIOGRAPHIE}

BUCKLAND M., "What is a digital document ", Document numérique, 1998, vol. $2, \mathrm{n}^{\circ} 2$.

CHABIN A.-M., « Document-trace et document source. La technologie numérique change-t-elle la notion de document ? ", Information-Interaction-Intelligence, 2004, vol. $4, n^{\circ} 1$.

CHANDLER A. D., La main visible du manager, Paris, Economica, 1988.

FOUCAULT M., Surveiller et punir, Paris, Gallimard, 1975.

GARDEY D., Ecrire, compter, classer : comment une révolution de papier a transformé les sociétés contemporaines, 1800-1940, Paris, La Découverte, 2008.

GOODY J., La raison graphique, la domestication de la pensée sauvage, Paris, Minuit, 1979.

GOODY J., La logique de l'ecriture, Paris, Armand Colin, 1986.

JACOB C., "La Leçon d'Alexandrie ", Autrement, 1991, n¹21, p. 23-32.

JEANNENEY J.-N., Quand Google défie l'Europe, Paris, Mille et une nuits, 2006.

JEANNERET Y., SOUCHIER E., « Ecriture numérique ou médias informatisés ? », Pour la Science, Dossier hors-série Du signe à l'écriture, octobre-janvier 2002.

LATOUR B., La science en action, Paris, La Découverte, 1989.

MERZEAU L., " Du signe à la trace : l'information sur mesure ", Hermès, 2009, n 53 , p. 23-29.

MERZEAU L., « Mémoire », Medium, n 9, 2006.

PINÈDE N., « Logiques et stratégies de pouvoir dans les organisations via les TIC : ruptures et/ou continuités ? », Colloque SFSIC, Rennes, 2006.

POIROT-DELPECH S. L., GRAS A., Grandeur et dépendance : sociologie des macro-systèmes techniques, Paris, PUF, 1993.

ROBERT P., «Entre critique et modélisation,pour une « nouvelle posture critique » face à l'informatisation ", colloque du CREIS, 2004.

ROBERT P., La logique politique des technologies de l'information, critique de la logistique du "glissement de la prérogative politique ", Bordeaux, Presses universitaires de Bordeaux, (collection Labyrinthes), 2005.

ROBERT P., Une théorie sociétale des TIC, Paris, Hermès, 2009. 
ROBERT P., Mnémotechnologies, pour une théorie générale critique des technologies intellectuelles, Paris, Hermès, 2010.

ROBERT P., « Critique de la gestionnarisation », Bruxelles, colloque EUTIC, 2012.

RTP-DOC, Le texte en jeu, permanence et transformations du document, Paris, CNRS/ Enssib, version 2004.

RTP-DOC, Document et modernités, Paris, CNRS/Enssib, 2006.

SOUCHIER E., « L'image du texte, pour une théorie de l'énonciation éditoriale », Les Cabiers de médiologie, n 6, 1998.

SOUCHIERE., «L'écrit d'écran, pratiques d'écriture \&informatique», Communication et langages, $\mathrm{n}^{\circ} 107,1^{\mathrm{er}}$ trimestre 1996, p. 105-119.

Résumé : Nous explorons dans ce texte quelques pistes à propos de l'impact du numérique sur le statut du document. Nous revenons d'abord sur les différences entre les modes d'existence traditionnel et numérique du document. Puis nous voyons en quoi les nouveaux effets de décontextualisation générés par le numérique influent sur les rapports que nouent les processus logistiques et documentaires. Ce qui n'est pas sans conséquences sur les plans politique et organisationnel.

Mots-clés : Décontextualisation, dématérialisation, document numérique, logistique, mémoire sociale.

Abstract : We investigate in this paper some ideas about the impact of digital technology on the status of document. We consider first the differences between traditional and digital documents. Then we see how new effects generated by the digital decontextualization affect relations between logistics and documentaries processes, which is not without consequences on a political and organizational point of view.

Keywords : Decontextualization, Dematerialization, Digital document, Logistics, Social memory. 
